\title{
Staphylococcus aureus and Coagulase-Negative Staphylococci in Bacteraemia: The Epidemiology, Predisposing Factors, Pathogenicity and Antimicrobial Resistance
}

\author{
John-Ugwuanya A Grace ${ }^{1 *}$, Busayo O Olayinka ${ }^{1}$, Josiah A Onaolapo ${ }^{1}$ and Stephen K Obaro ${ }^{2}$ \\ ${ }^{1}$ Department of Pharmaceutics and Pharmaceutical Microbiology, Ahmadu Bello University, Zaria, Kaduna, Nigeria \\ ${ }^{2}$ Division of Pediatric Infectious Disease University of Nebraska Medical Center, Omaha, Nebraska, United States
}

"Corresponding author: John-Ugwuanya A Grace, Department of Pharmaceutics and Pharmaceutical Microbiology, Ahmadu Bello University, Zaria, Kaduna, Nigeria, Tel: +2347061145614; E-mail: graceamy2k@yahoo.com

Received date: January 18, 2019; Accepted date: February 04, 2019; Published date: February 12, 2019

Copyright: ( 2019 Grace JA, et al. This is an open-access article distributed under the terms of the Creative Commons Attribution License, which permits unrestricted use, distribution, and reproduction in any medium, provided the original author and source are credited.

\section{Abstract}

Staphylococcus species are the predominant Gram-positive organisms obtained from blood culture samples. Its incidence in bloodstream infection among children and adults varies among. Staphylococcus aureus is regarded as pathogenic with high morbidity and mortality while coagulase-negative staphylococci (CoNS) are often regarded as a contaminant and not a true cause of bacteremia despite its rising occurrence. Predisposing factors of staphylococcal bacteremia include malnutrition, malaria, HIVIAIDS and nosocomial infections. Methicillin-resistance in Staphylococcus aureus and CoNS in bacteremia is associated with an increase in multidrug-resistant virulent strains when compared to methicillin-sensitive $S$. aureus or CoNS. The impact of coagulase-negative staphylococci in bacteremia is on the increase with marked clinical complications. However, this review summarizes the prevalence and epidemiology of $S$. aureus and CoNS in bacteremia based on research outcomes in Africa, Asia, Europe, and North America.

Keywords: Staphylococcus aureus, Coagulase-negative staphylococci; Bacteraemia

\section{Introduction}

Staphylococci species are members of the family Micrococcaceae known to be Gram-positive cocci occurring singly, in pairs, short chains and as rough grape-like clusters. They are catalase-positive and also differentiated based on their ability to produce coagulase, causing visible agglutination on slide and tube coagulase test [1].

Staphylococcus aureus bacteremia is defined as the microbiological presence of $S$. aureus in the bloodstream. It is characterized by high morbidity, mortality and associated with frequent use of intravascular devices, invasive surgeries and immunodeficiency in patients. It has become a leading cause of hospital and community-acquired bacteremia [2]. In intensive care units, $S$. aureus is the most common Gram-positive pathogen isolated in bloodstream infections [3].

Coagulase-negative staphylococci (CoNS) are found as normal flora on the skin and mucous membrane of humans [4]. It is commonly reported in very-low-birth-weight neonates, immune-compromised patients, and individuals with indwelling intravascular devices or implanted medical devices [5]. Being a commensal on the human skin, CoNS can be transmitted from the hands of medical staff and caregivers especially in children [6].

In neonates, CoNS accounts for about $70 \%$ of the positive blood culture, although they are mostly regarded as contaminants [7]. True bacteremia with CoNS occurs mostly in preterm neonates below 32 weeks gestation based on their under-developed immunity. Obolski et al. pointed out that before CoNS are considered as contaminants, they should be tested for resistance [8].
Clinical diagnosis of CoNS bacteremia involves a positive blood culture, a medical picture of health deterioration and an acute phase inflammatory marker usually C-reactive protein or two positive CoNS blood cultures collected consecutively within 48 hours or a positive blood culture in mixed growth with significant pathogenic bacteria $[7,9]$. In a study by Elzi et al., the clinical significance of CoNS as a true pathogen using a single positive blood culture was evaluated based on the presence of a central venous catheter and two Systemic Inflammatory Response Syndromes (SIRS) or at least three SIRS [10]. Some reports from sub-Saharan Africa brings to light that CoNS bacteremia is having an upsurge in occurrence and clinical significance [11].

Some study assumes that the high incidence of CoNS is due to the usage of $70 \%$ alcohol alone in skin cleaning before venipuncture instead of in combination with $10 \%$ povidone iodine [12]. Archibald et al. indicated an increased contamination rate with povidone-iodine [11].

\section{DISCUSSION}

\section{Epidemiology of staphylococci colonization}

Staphylococcus aureus can be obtained from human and animal hosts. It is highly clonal in nature with variants that can colonize specific or multiple hosts [13]. Molecular typing aids in the understanding of $S$. aureus epidemiology. Coagulase-negative staphylococci species are normal flora on the skin. Differentiating CoNS bacteremia infection from contamination in blood culture specimen poses some difficulty since CoNS is a widespread skin commensal [5]. There are about 38 recognized CoNS species with 13 colonizing humans e.g. Staphylococcus saprophyticus, Staphylococcus 
Citation: Grace JA, Olayinka BO, Onaolapo JA, Obaro SK (2019) Staphylococcus aureus and Coagulase-Negative Staphylococci in Bacteraemia: The Epidemiology, Predisposing Factors, Pathogenicity and Antimicrobial Resistance. Clin Microbiol 8: 325. doi: $10.4172 / 2327-5073.1000325$

Page 2 of 5

haemolyticus, Staphylococcus hominis, Staphylococcus warneri, Staphylococcus xylosus, Staphylococcus epidermidis, Staphylococcus schlefieri, and Staphylococcus lugdunensis [14]. The common CoNS species isolated clinically and often considered for its virulence and pathogenicity is Staphylococcus epidermidis $[5,15,16]$.

A study on establishing the significance of bacteremia in 170 Ethiopian children with severe malnutrition showed that about $20.6 \%$ of the children yielded bacterial isolates with $S$. aureus $(28.6 \%)$ and coagulase-negative staphylococci $(22.9 \%)$ having a high frequency among other isolates (Figure 1). The finding indicates that CoNS species are posing great health problems and it is consistent with the statement by von Eiff et al, that the fraction of bloodstream infections caused by CoNS and the total incidence of true CoNS bacteremia are increasing [17].

Most bloodstream infection yielding coagulase-negative staphylococci are often regarded as contaminants despite their predominance as Gram-positive isolates in blood culture. A study on bacteremia among 140 Gambian children aged under 5 years with severe acute malnutrition showed that out of the 38 positive blood culture isolates obtained, CoNS yielded $50 \%$ and $S$. aureus $0 \%$ apart from other Gram-positive and negative bacterial isolates observed [18]. Despite the high percentage of CoNS, they were regarded as contaminants resulting in no further species identification and antibiotic sensitivity analysis. But a study by Onipede et al. considered CoNS as a true isolate in bacteremia and did antimicrobial susceptibility testing, regardless of the low percentage of CoNS (0.5\%) in the blood cultures [19].

This varies from the study by Obolski et al., where CoNS (31.5\%) was tested for antibiotic resistance before being regarded as contaminants [8]. Staphylococcus aureus yielded $4.2 \%$ among other significant bacteria. Their findings on CoNS resistance suggested that it could predict antibiotic resistance in subsequent bacteremia with a known pathogen, affect the mortality rate and also guide clinicians in their choice of antibiotic therapy.

A collaborative retrospective study was conducted on blood culture samples from different hospitals in Tanzania, Malawi, and the United States. Out of 54 blood cultures obtained in a medical facility in the United States that were positive for bacterial growth, CoNS and $S$. aureus yielded $44.4 \%$ and $27.8 \%$ respectively; although, CoNS was regarded as contaminants [11]. High aseptic standard maintained during venipuncture and blood culture inoculation in the study at Tanzania resulted in decreased contaminant, with $S$. aureus and CoNS occurring as $2.5 \%$ and $0.6 \%$ respectively [20]. The study in Malawi had CoNS, specifically $S$. epidermidis as $0.4 \%$, which was indicated as contaminants [21].

The detailed study on staphylococcal isolates carried out in 6 healthcare facilities in Ghana indicated that about $12.4 \%$ were $S$. aureus from Bloodstream Infections (BSI) while 65.9\% signifying coagulase-negative staphylococci originated from bacteremia, skin and soft tissue infection (SSTI) and other infections [22]. Elzi et al. assessed the clinical importance of CoNS bacteremia using the Systemic Inflammatory Response Syndrome (SIRS) with 35\% identified as true bacteremia out of 654 CoNS isolates [10]. The SIRS criteria used include temperature $>38^{\circ} \mathrm{C}$ or $<36^{\circ} \mathrm{C}$, respiratory rate $>20 / \mathrm{min}$, heart rate $>90 / \mathrm{min}$ and white blood cell count $>12 \mathrm{G} / \mathrm{L}$ or $<4 \mathrm{G} / \mathrm{L}$.

\section{Predisposing factors}

Malnutrition in sub-Saharan African and South Asia is one of the widespread causes of childhood morbidity and mortality, with severe acute malnutrition affecting roughly one-tenth of children under 5 years globally. Resultant death of over $50 \%$ is associated with children living in abject poverty and unbalanced diet, and disease prognosis worsens with HIV/AIDS infection [4]. Growth retardation, muscle wastage and weight loss are common features of childhood malnutrition. Susceptibility to infection increased due to lowered immune defense mechanism and often makes clinical diagnosis and treatment difficult by concealing symptoms of other infectious diseases.

In Ethiopia, about one in two children below five years have stunted growth, muscle wastage and reduced weight for age especially among children in rural areas [4]. Staphylococcal species were the main cause of bacteremia in the malnourished children with CoNS species occurrence being possibly linked to immune suppression in severely malnourished children. Standard use of antibiotics for empirical therapy is proposed by WHO for children with severe acute malnutrition since they are predisposed to bacteremia, diarrhea, urinary tract infection and pneumonia [18].

Malaria in African children below 5 years is about $90 \%$ on a scale of approximately 500 million cases reported yearly globally. The medical presentation of malaria is closely similar to septicemia but having limited local data on its correlation [23]. A study in Nairobi on admitted febrile children with malaria in the age range of 3 months to 12 years indicated that 18 out of 158 children with malaria had bacteremia while 14 out of 106 had bacteremia but without malaria. Coagulase-negative staphylococci (34.4\%) were the predominant Gram-positive bacteria but were regarded as contaminants while Staphylococcus aureus yielded 12.5\% [23]. However, co-infection of bacteremia with or without malaria appeared to be similar in this study occurring as $11.4 \%$ and $13.2 \%$ respectively.

Nosocomial infections, which is particularly common in intensive care units are often associated with $S$. aureus bacteremia [5]. It is characterized by high virulence with a mortality rate between $20 \%-50 \%$ (2). Nosocomial $S$. aureus bacteremia is often considered if it appears after 72 hours of hospital admission.

The German nosocomial infection surveillance system (KISS) reported that $S$. aureus yields about $16.3 \%$ of the nosocomial infections in the intensive care unit [24]. Methicillin-Resistant $S$. aureus (MRSA) was observed in about $42.9 \%$ of hospital-acquired bloodstream infection when compared to $7.9 \%$ community-acquired MRSA [12]

Recent trends have revealed the importance of CoNS infection as nosocomial pathogens, depicted by the significant rise in morbidity, mortality and healthcare cost in pediatric and neonatal care unit [25]. The study by Blot et al., showed that $0.38 \%$ were diagnosed with $S$. aureus bacteremia [2]. Approximately $17.6 \%$ of the admitted patients developed $S$. aureus bacteremia within 72 hours and did not have their main diagnosis as septicemia at the time of admission. 
Citation: Grace JA, Olayinka BO, Onaolapo JA, Obaro SK (2019) Staphylococcus aureus and Coagulase-Negative Staphylococci in Bacteraemia: The Epidemiology, Predisposing Factors, Pathogenicity and Antimicrobial Resistance. Clin Microbiol 8: 325. doi: $10.4172 / 2327-5073.1000325$

Page 3 of 5

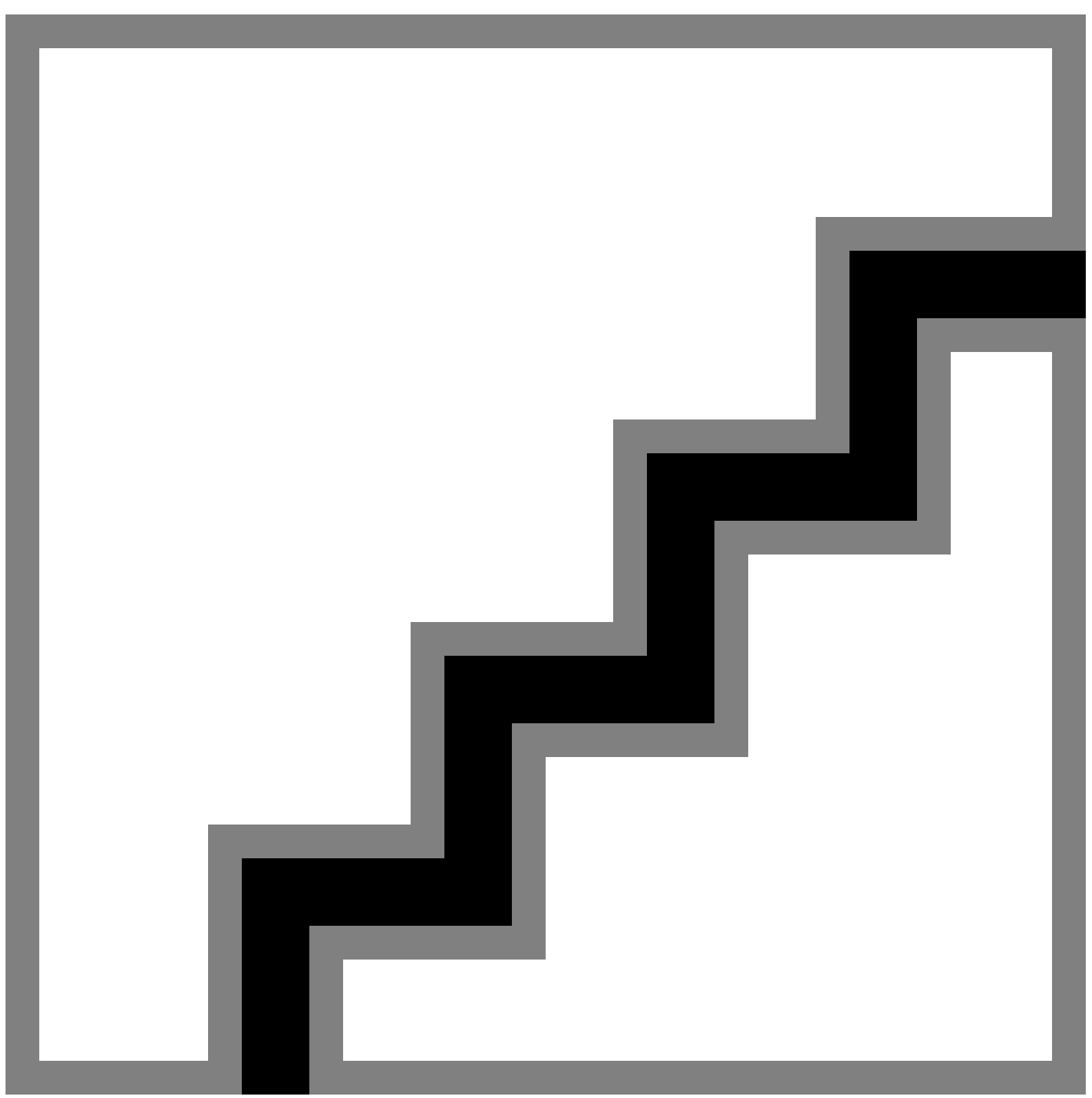

Figure 1: Frequency of $S$. aureus and CoNS in bacteremia.

\section{Pathogenicity and virulence}

Staphylococcal species have different classes of surface proteins based on their structural and functional differences. These proteins are covalently bound to the peptidoglycan layer of the cell wall. It is an important virulence factor necessary for their survival as commensals or pathogens [26]. The functions of surface proteins include cell adhesion, invasion, and colonization of host tissues, evading of the immune response and production of slime that aids their adherence to indwelling medical devices such as valvular prostheses, central and peripheral venous catheters, artificial heart valves, pacemakers, and orthopedic prosthesis. CoNS produce proteases, lipases and other exoenzymes that enable it to thrive and possibly degrade host tissues [27].
Staphylococcus aureus produces a vital and unique virulence factor known as thermostable nuclease (TNase), which is encoded by the nuc gene [28]. Conversely, other coagulase-negative staphylococci strains such as $S$. simulans, $S$. caprae, $S$. intermidus, $S$. carnosus, and $S$. capitis produce TNase but can be differentiated from TNase produced by $S$. aureus using the monoclonal antibody and nuclease-based test [29].

It is important to note that most MSSA isolates from Africa harbor the Panton-Valentine Leucocidin (PVL) genes: lukS- $p v$ and $l u k F-p v$, about $55 \%$ more than the rest of the world [22]. This may be linked to the interrelationship between the host, the microorganism and the environment [30]. The genetic material of a bacteriophage that infects $S$. aureus produces the PVL, thereby making it more virulent. A study on $S$. aureus isolates indicates that there are some genotypes and virulence factors that are widespread in Africa due to staphylococcal 
Citation: Grace JA, Olayinka BO, Onaolapo JA, Obaro SK (2019) Staphylococcus aureus and Coagulase-Negative Staphylococci in Bacteraemia: The Epidemiology, Predisposing Factors, Pathogenicity and Antimicrobial Resistance. Clin Microbiol 8: 325. doi: $10.4172 / 2327-5073.1000325$

Page 4 of 5

infection than from colonization; e.g. PVL encoded by the lukS-pvand lukF-pvgenes [31].

In a study by Giormezis et al, CoNS isolates were tested for antimicrobial susceptibility, biofilm formation, $\operatorname{mec} A$, ica operon, adhesin (accumulation-associated protein aap and biofilm-associated protein bap) and toxin genes [32]. Approximately 58\% produced biofilm; 7\% carried the ica operon which encodes the production of polysaccharide intercellular adhesin; 30\% carried the adhesin genes while $99 \%$ carried the mecA gene and showed significant multidrug resistance.

Among the CoNS species, Staphylococcus epidermidis has had more research conducted on virulence factors such as biofilm formation, adhesion and aggregation. Adherence of CoNS to the host skin or indwelling medical device is the first step to colonization or infection [5]. This can occur directly or by means of extracellular matrix components and the host plasma protein components, specifically collagen, fibronectin, fibrinogen and heparin-related polysaccharides. Microbial surface components recognizing adhesive matrix molecules (MSCRAMMs) are adhesins expressed on bacteria cell surface that mediates adherence to the host [32].

Biofilm formation is an important factor in the pathogenesis of staphylococcal infections, which confers increased metabolic, mechanical, immune and antibiotic resistance. It is a microbial-derived sessile community comprising cells which are irreversibly attached to an interface or each other and embedded into the extracellular polymeric matrix that they produce [32-34]. The production of staphylococcal toxin such as enterotoxins and toxic shock syndrome, which act as superantigens toxin are vital to its pathogenesis. They bind the major histocompatibility complex class II directly, thereby triggering an increase in the T-cell stimulation and excess production of cytokine [32].

\section{Antimicrobial resistance}

The main public health problem associated with high mortality, longer hospitalization, and exorbitant treatment cost is antibiotic resistance. For effective analysis of bacteremia, the collection of blood cultures should precede empirical treatment and modified based on antibiotic susceptibility and resistance pattern [8]. Bacteremia caused by methicillin-resistant $S$. aureus results in greater mortality with an increased tendency of resistance to various antimicrobial agents when compared to Methicillin-sensitive $S$. aureus (MSSA) bacteremia [35]. The study by Gastmeier, et al., showed increased mortality [24] with MRSA (16.8\%) when compared with MSSA (6.0\%). It also results in increased healthcare cost with limited options for treatment due to multiple drug resistance [36].

Methicillin resistance depicted by the presence of the $\operatorname{mec} A$ gene in $S$. aureus and CoNS varies between different studies. This gene encodes the penicillin-binding protein with decreased affinity to $\beta$ lactam antibiotics [32].

In Africa, antimicrobial resistance in $S$. aureus is emerging, specifically methicillin-resistance in varying genetic backgrounds [30]. This genetic diversity has led to the emergence of 6 major clones globally with rising spread across to Africa. The clones are referred to as staphylococcal cassette chromosome SCCmec type I-VI [37]. A survey on bacteremia in South Africa reported about 23\% prevalence of MRSA among the $S$. aureus isolates [36]. The majority of CoNS isolates have the mecA gene and an increase in multidrug-resistant virulent CoNS strains causing difficulty to treatment [32].
Vancomycin, a glycopeptide antibiotic is used as the main therapy but has shown resistance [5]. A typical African MRSA clone observed in some studies is the ST88-MRSA-III/IV.

Although a retrospective study by Blot et al., was carried out on critically ill adults aged between 49 to 70 years within a 7 -year period in a Belgium hospital (2), the study showed that the mortality rate in patients with MRSA bacteremia was high (63.8\%) when compared to MSSA (23.7\%). However, the patients with MRSA bacteremia were elderly, sickly and more debilitated. There are conflicting data in comparing MRSA and MSSA bacteremia with varying effect on fatality rate.

A common feature in staphylococcal research in Africa is the high level of resistance to penicillin among other antimicrobial agents; this is encoded by the bla $Z$ gene [31]. According to a study by Alem et al., all the $S$. aureus and CoNS isolates obtained showed resistance to penicillin, oxacillin, ampicillin, and chloramphenicol. Few of the isolates also showed resistance to erythromycin, tetracycline, ciprofloxacin, cotrimoxazole, vancomycin, and ceftriaxone in varying proportion [30]. This agrees with the high percentage of multi-drug resistant $S$. aureus observed by Onipede et al. [19].

\section{Conclusion}

Staphylococcal isolates, especially $S$. aureus are a major cause of bacteremia in malnourished children. The impact of CoNS in bloodstream infection is rising with major health complications, thereby indicating its possibility of clinical significance in bacteremia. The need to identify the CoNS species and determine their pathogenic potential instead of totally disregarding them as contaminants will give a better understanding of their role in true bacteremia and also aid clinical management.

\section{References}

1. Feltham RK (2008) A taxonomic study of the Micrococcaceae. J Appl Microbiol 47: 243-254.

2. Blot SI, Vandewoude KH, Hoste EA, Colardyn FA (2002) Outcome and attributable mortality in critically ill patients with bacteremia involving methicillin-susceptible and methicillin-resistant Staphylococcus aureus. Archives of Intern Med 162: 2229-2235.

3. García-Vázquez E, Fernández-Rufete A, Hernández-Torres A, Canteras M, Ruiz J, et al. (2013) When is coagulase-negative Staphylococcus bacteremia clinically significant? Scand J Infect Dis 45: 664-671.

4. Alem A, Abdissa A, Beyene G, Getahun G, Girma T (2011) Bacteraemia among severely malnourished children in Jimma Hospital, Ethiopia. Ethiop J Health Sci 21: 175-182.

5. Venkatesh MP, Placencia F, Weisman LE (2006) Coagulase-negative Staphylococcal infections in the neonate and child: An update. Seminars on Pediatric Infect Dis 17: 120-127.

6. Remington JS, Klein JO, Wilson BC, Nizet V, Maldonado YA (2011) Staphylococcal infections. Infect Dis of the Fetus and Newborn pp: 489-505.

7. Modi N, Dore CJ, Saraswatula A, Richards M, Bamford KB, et al. (2009) A case definition for national and international neonatal bloodstream infection surveillance. Arch Dis Child Educ Pract Ed 94: F8-F12.

8. Obolski U, Alon D, Hadany L, Stein GY (2014) Resistance profiles of coagulase-negative staphylococci contaminating blood cultures predict pathogen resistance and patient mortality. J Antimicrobiol Chemotherapy 69: 2541-2546.

9. John JF, Davidson RJ, Low DE (2015) Staphylococcus epidermidis and other coagulase-negative staphylococci. Infectious Disease Antimicrobial Agents 45: 293-298. 
Citation: Grace JA, Olayinka BO, Onaolapo JA, Obaro SK (2019) Staphylococcus aureus and Coagulase-Negative Staphylococci in Bacteraemia: The Epidemiology, Predisposing Factors, Pathogenicity and Antimicrobial Resistance. Clin Microbiol 8: 325 . doi: 10.4172/2327-5073.1000325

Page 5 of 5

10. Elzi L, Babouee E, Vogeli N, Laffer R, Dangel M, et al. (2012) How to discriminate contamination from bloodstream infection due to coagulase-negative staphylococci: a prospective study with 654 patients. Clin Microbiol Infect 18: 355-361.

11. Archibald LK, Pallangyo K, Kazembe P, Reller BL (2006) Blood culture contamination in Tanzania, Malawi and the United States: A microbiological tale of three cities. J Clin Microbiol 44: 4425-4429.

12. Stoesser N, Moore CE, Pocock JM, An KP, Emary K, et al. (2013) Pediatric bloodstream infections in Cambodia, 2007 to 2011. The Pediatric Infect Dis Journal 32: e272-e276.

13. Akobi B, Aboderin O, Sasaki T, Shittu A (2012) Characterisation of Staphylococcus aureus isolates from faecal samples of the straw-colored fruit bat (Eidolon helvum) in Obafemi Awolowo University (OAU), Nigeria. BioMed Central Microbio 12: 1-8.

14. Cunha MR, Calsolari RO (2007) Toxigenicity in Staphylococcus with emphasis on coagulase-negative staphylococci. Communicating Current Research and Educational Topics and Trends in Applied Microbiology 2007: 778-782.

15. Qin L, Da F, Fisher EL, Tan DC, Nguyen TH, et al. (2017) Toxin mediates sepsis caused by methicillin-resistant Staphylococcus epidermidis. PLoS Pathogens 13: e1006153.

16. Koksal F, Yasar H, Samasti M (2009) Antibiotic resistance patterns of coagulase-negative staphylococcus strains isolated from blood cultures of septicemic patients in Turkey. Microbiol Res 164: 404-410.

17. Eiff C, Peters G, Heilmann C (2002) Pathogenesis of infections due to coagulase-negative staphylococci. Lancet Infect Dis 2: 677-685.

18. Okomo AU, Garba D, Fombah AE, Secka O, Ikumapayi UN, et al. (2011) Bacterial isolates and antibiotic sensitivity among Gambian Children with severe acute malnutrition. Intern Journal of Pediatrics 23: 1-8.

19. Onipede AO, Onayade AA, Elusiyan JBE, Obiajunwa PO, Ogundare EOO, et al. (2009) Invasive bacterial isolates from children with severe infections in a Nigerian hospital. J Infect in Developing Countries 3: 429-436.

20. Archibald LK, Dulk DMO, Pallangyo KJ, Reller LB (1998) Fatal Mycobacterium tuberculosis bloodstream infections in febrile hospitalized adults in Dar es Salaam, Tanzania. Clin Infect Dis 26: 290-296.

21. Archibald LK, McDonald LC, Nwanyanwu O, Kazembe P, Dobbie T, et al. (2000) A hospital-based prevalence survey of bloodstream infections in febrile patients in Malawi: implications for diagnosis and therapy. J Infect Dis 81: 1414-1420.

22. Egyir B, Guardabassi L, Sorum M, Nielsen SS, Kolekang A, et al. (2014) Molecular epidemiology and antimicrobial susceptibility of clinical Staphylococcus aureus from healthcare institutions in Ghana. PLoS ONE 9: 1-7.

23. Okwara FN, Obimbo EM, Wafula EM, Murila FV (2004) Bacteraemia, urinary tract infection and malaria in hospitalized febrile children in Nairobi: Is there an association. East African Med Jour 81: 47-51.

24. Gastmeier P, Sohr D, Geffers C, Behnke M, Daschner F, et al. (2005) Mortality risk factors with nosocomial Staphylococcus aureus infections in intensive care units: Results from the German nosocomial infection surveillance system (KISS). Infect 33: 50-55.
25. De-Silva GD, Justice A, Wilkinson AR, Buttery J, Herbert MD, et al. (2001) Genetic population structure of coagulase-negative staphylococci associated with carriage and disease in preterm infants. Clin Infect Dis 33: $1520-1528$

26. Foster TJ, Geoghegan JA, Ganesh VK, Hook M (2014) Adhesion, invasion and evasion: the many functions of the surface proteins of Staphylococcus aureus. Nat Rev Microbiol 12: 49-62.

27. Azih A, Enabulele I (2013) Species distribution and virulence factors of coagulase-negative staphylococci isolated in clinical samples from the University of Benin Teaching Hospital, Edo State, Nigeria. J Natural Sci Res 3: 38-43.

28. Hu Y, Xie Y, Tang J, Shi X (2012) Comparative expression analysis of two thermostable nuclease genes in Staphylococcus aureus. Foodborne Pathogens and Dis 9: 265-271.

29. Brakstad OG, Maeland JA, Chesneau O (1995) Comparison of tests designed to identify Staphylococcus aureus thermostable nuclease. Apmis 103: 219-224.

30. Schaumburg F, Alabi AS, Peters G, Becker K (2014) New Epidemiology of Staphylococcus aureus infection in Africa. Clinical Microbiology and Infection 20: 589-596.

31. Ayepola OO, Olasupo NA, Egwari LO, Becker K, Schaumburg F (2015) Molecular characterization and antimicrobial susceptibility of Staphylococcus aureus isolated from clinical infection and asymptomatic carriers in Southwest Nigeria. PLoS ONE 10: 1-8.

32. Giormezis N, Kolonitsiou F, Foka A, Drougka E, Liakopoulos A, et al. (2014) Coagulase-negative Staphylococcal bloodstream and prostheticdevice-associated infections: The role of biofilm formation and distribution of adhesion and toxin genes. Journal of Medical Microbiology 63: 1500-1508.

33. Diekema DJ, Pfaller MA, Schmitz FJ, Smayevsky J, Bel J, et al. (2001) Survey of infections due to Staphylococcus species: Frequency and antimicrobial susceptibility of isolates collected in the United States, Canada, Latin America, Europe and Western Pacific region for the SENTRY antimicrobial surveillance program, 1997-1999. Clinical Infectious Diseases 32: S114-132.

34. Dong Y, Speer CP, Glaser K (2018) Beyond sepsis: Staphylococcus epidermidis is an underestimated but significant contributor to neonatal morbidity. Virulence 9: 621633.

35. Fortuin-de Smidt MC, Singh-Moodley A, Badat R, Quan V, Kularatne R, et al. (2015) Staphylococcus aureus bacteremia in Guateng academic hospitals, South Africa. International Journal of Infectious Diseases 30: 41-48.

36. Moodley A, Oosthuysen WF, Duse AG, Marais E (2010) South African MRSA Surveillance Group. Molecular characterization of clinical methicillin-resistant Staphylococcus aureus isolates in South Africa. J Clin Microbiol 48: 4608-4611.

37. Falagas ME, Karageorgopoulos DE, Leptidis J, Korbila IP (2013) MRSA in Africa: Filling the global map of antimicrobial resistance. PLoS ONE 8: e68024. 